\title{
Single-chain Fv phage display propensity exhibits strong positive correlation with overall expression levels Nathan Scott ${ }^{3}$, Catherine B Reynolds ${ }^{3}$, Michael J Wright ${ }^{1,3}$, Omar Qazi ${ }^{2,3}$, Neil Fairweather ${ }^{3}$ and Mahendra P Deonarain*3
}

Address: ${ }^{1}$ UCB-Celltech, 208 Bath Road, Slough, Berkshire, SL1 3WE, UK, ${ }^{2}$ Department of Paediatrics, University of Texas Medical Branch (UTMB), Galveston, TX 77555, USA and ${ }^{3}$ Department of Life Sciences, Faculty of Natural Sciences, Imperial College London, Exhibition Road, London, SW7 2AZ, UK

Email: Nathan Scott - nathan.scott04@imperial.ac.uk; Catherine B Reynolds - catherine.reynolds06@imperial.ac.uk; Michael J Wright - Michael.Wright@ucb-group.com; Omar Qazi - o.qazi@imperial.ac.uk; Neil Fairweather - n.fairweather@imperial.ac.uk; Mahendra P Deonarain* - m.deonarain@imperial.ac.uk

* Corresponding author

Published: 29 December 2008

BMC Biotechnology 2008, 8:97 doi:10.1/86/1472-6750-8-97
Received: 23 July 2008

Accepted: 29 December 2008

This article is available from: http://www.biomedcentral.com/1472-6750/8/97

(C) 2008 Scott et al; licensee BioMed Central Ltd.

This is an Open Access article distributed under the terms of the Creative Commons Attribution License (http://creativecommons.org/licenses/by/2.0), which permits unrestricted use, distribution, and reproduction in any medium, provided the original work is properly cited.

\begin{abstract}
Background: Single chain Fvs (scFvs) are widely applied in research, diagnostics and therapeutic settings. Display and selection from combinatorial libraries is the main route to their discovery and many factors influence the success of this process. They exhibit low thermodynamic stability, resulting in low levels of premature cytosolic folding or aggregation which facilitates sec YEG-mediated translocation and phage in $E$. coli. However, there is little data analysing how this is related to and influenced by scFv protein expression.

Results: We characterised the relationship between overall scFv expression and display propensity for a panel of 15 anti-tetanus toxin scFvs and found a strong positive correlation (Rho $=0.88, p<0.005$ ) between the two parameters. Display propensity, overall expression and soluble localisation to the periplasm and extracellular fractions were clone specific characteristics which varied despite high levels of sequence homology. There was no correlation between display of scFv or its expression in non-fused (free) form with soluble scFv localisation to the periplasm or culture supernatant. This suggests that divergence in the fate of scFv-plll and non-fused scFv after translocation to the periplasm accounts for the observed disparity. Differential degrees of periplasmic aggregation of non-fused scFv between clones may affect the partitioning of scFv in the periplasm and culture supernatant abrogating any correlation. We suggest that these factors do not apply to the scFv-plll fusion since it remains anchored to the bacterial inner membrane as part of the innate phage packaging and budding process.
\end{abstract}

Conclusion: We conclude that in the absence of premature cytosolic aggregation or folding, the propensity of a scFv to be displayed on phage is directly related to its overall expression level and is thus indirectly influenced by factors such as codon bias, mRNA abundance or putative DNA motifs affecting expression. This suggests that scFvs capable of high overall expression and display levels may not produce high yields of non phage-fused soluble protein in either the periplasmic or extracellular fractions of $E$. coli. This should be considered when screening clones selected from combinatorial libraries for further study.

The nucleotide and amino acid sequences of the anti-tetanus toxin scFvs have been deposited in the EMBL data base: accession numbers-CI: AM749134, C2: AM749135, C3: AM749136, C4: AM749137, C5: AM749138, NI: AM749139, N2: AM749I40, N3: AM749|4I, N4: AM749I42, N5: AM749I43 JI; AM749I44, J2: AM749I45, J3: AM749I46, J4: AM749I47, J5: AM749I48. 


\section{Background}

During the last two decades single chain $\mathrm{Fv}(\mathrm{scFv})$ antibodies have become widely applied in research, diagnostics and therapeutic settings [1]. These recombinant, antigen-binding molecules can be engineered $[2,3]$ to modulate their specificity [4] affinity [5] and pharmacokinetics [6] as well as appending novel effector functions $[7,8]$ Established technology allows investigators to produce large and diverse combinatorial scFv libraries commonly using minor coat protein (pIII) filamentous phage display in Escherichia coli (E. coli) [9-12]. During production of phage-scFvs, the scFv-pIII fusion is translocated to the periplasmic space and remains anchored in the cytosolic membrane by the C-terminal hydrophobic extension of PIII [13]. The fusion protein then assembles with nascent phage particles as they extrude from the inner membrane.

Overall levels of scFv expression, as with all proteins, are dependant on transcriptional, post-transcriptional and translational level gene regulation. It has been shown that $47 \%$ of the variation in E. coli protein abundance is accounted for by mRNA abundance alone and codon-bias and codon adaptation indices account for a major proportion of the remaining variation $[14,15]$. Expression yield often refers to the level of soluble protein produced in the E. coli which may be located in the periplasm or in culture supernatant. High thermodynamic stability, high molecular weight, increased hydrophobicity and areas of low sequence complexity are linked to poor soluble protein expression yields [16]. Such properties can lead to proteins being more susceptible to proteolytic degradation [17] aggregation and inclusion body formation in either the cytosol [18] or periplasmic space [19,20]. Disulphiderich proteins may also be prone to mis-folding once in the periplasm [21].

There is a multitude of research demonstrating that improvements in soluble expression yields in either the periplasm or supernatant can typically be gained by approaches including removal of detrimental hydrophobic residues $[22,23]$, alteration of leader sequence [24], co-expression or over-expression of cytosolic or periplasmic chaperones $[25,26]$ or modifying induction conditions such as inducer concentration, temperature or time $[27,28]$.

Some processes affecting soluble protein expression yield also have bearing upon phage display. In phagemid vector systems [29] the protein-pIII fusion is targeted to the periplasm in the same manner as non-fused protein as they share the same leader sequence. Proteins refractory to SecYEG-mediated periplasmic translocation therefore tend to display poorly in standard phage display systems, elegantly shown for DARPins which have very high ther- modynamic stability and are prone to premature folding and aggregation in the cytosol post-translationally [30]. Display levels and periplasmic localisation were drastically improved when the leader sequence was altered for utilisation of the co-translational translocation signal recognition particle pathway which does not allow premature folding or aggregation. This study concluded that when comparing a diverse range of proteins, overall expression level may not correlate with phage display propensity but soluble periplasmic levels do.

There is little data available indicating how overall protein expression level relates to display propensity in the absence of dysfunctional cytosolic-periplasmic protein translocation. The $\mathrm{scFv}$, which is known for its lower thermodynamic stability [31] tends not to prematurely fold in the cytosol of bacteria and is amenable to display and localisation to the periplasm regardless of whether a post or co-translational translocation approach is used [30,32]. Comparing a panel of scFvs in terms of overall protein expression and phage display propensity allows the link between the two characteristics to be investigated.

We used phage display to select scFvs against the recombinant C-terminal domain of the tetanus toxin heavy chain $\left(\mathrm{H}_{\mathrm{C}}\right)$ (Scott, N. et al, manuscript in preparation) from a Cambridge Antibody Technology library containing $1.4 \times 10^{10}$ clones [29]. After two rounds of display we isolated many $\mathrm{H}_{\mathrm{C}}$-specific clones exhibiting complimentarity determining region (CDR) diversity (Scott et al, manuscript in preparation). The scFvs consist of an N-terminal SecYEG translocon signal sequence (VKKLLFAIPLVVPFYAAQPAMA) [29] and C-terminal hexahistidine $\left(\mathrm{his}_{6}\right.$ ) and c-myc tags. We sought to compare the phage display properties of 15 anti-Hc scFvs (termed C1C5, N1-N5 and J1-J5 based on differential epitope binding) in the suppressor strain of E. coli XL1-Blue with their facility of expression as non-fused scFvs in the non-suppressor strain HB2151. We observed a strong level of positive correlation between relative overall non-fused $\mathrm{scFv}$ expression and relative display level propensity.

\section{Results and discussion Phage display propensity of scFvs is a variable and clone- specific characteristic}

Phage from E. coli XL1-Blue cultures harbouring 15 different anti-Hc scFvs were made, concentrated, purified and quantified by UV spectroscopy (data not shown). Equal numbers of phage particles were blotted onto nitrocellulose following electrophoretic separation. All phage-scFv fusions were probed with either a monoclonal anti-pIII IgG antibody (Figure 1a) or monoclonal anti-his ${ }_{6}$ IgG antibody (Figure 1b). The helper phage only control showed a dominant band migrating at approximately 70 $\mathrm{kDa}$ with a variety of commonly observed smaller MW 


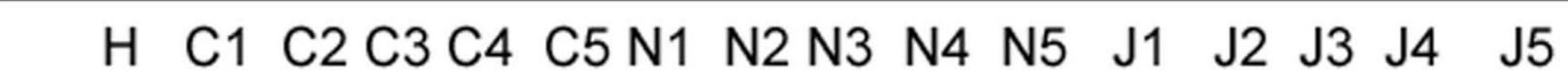
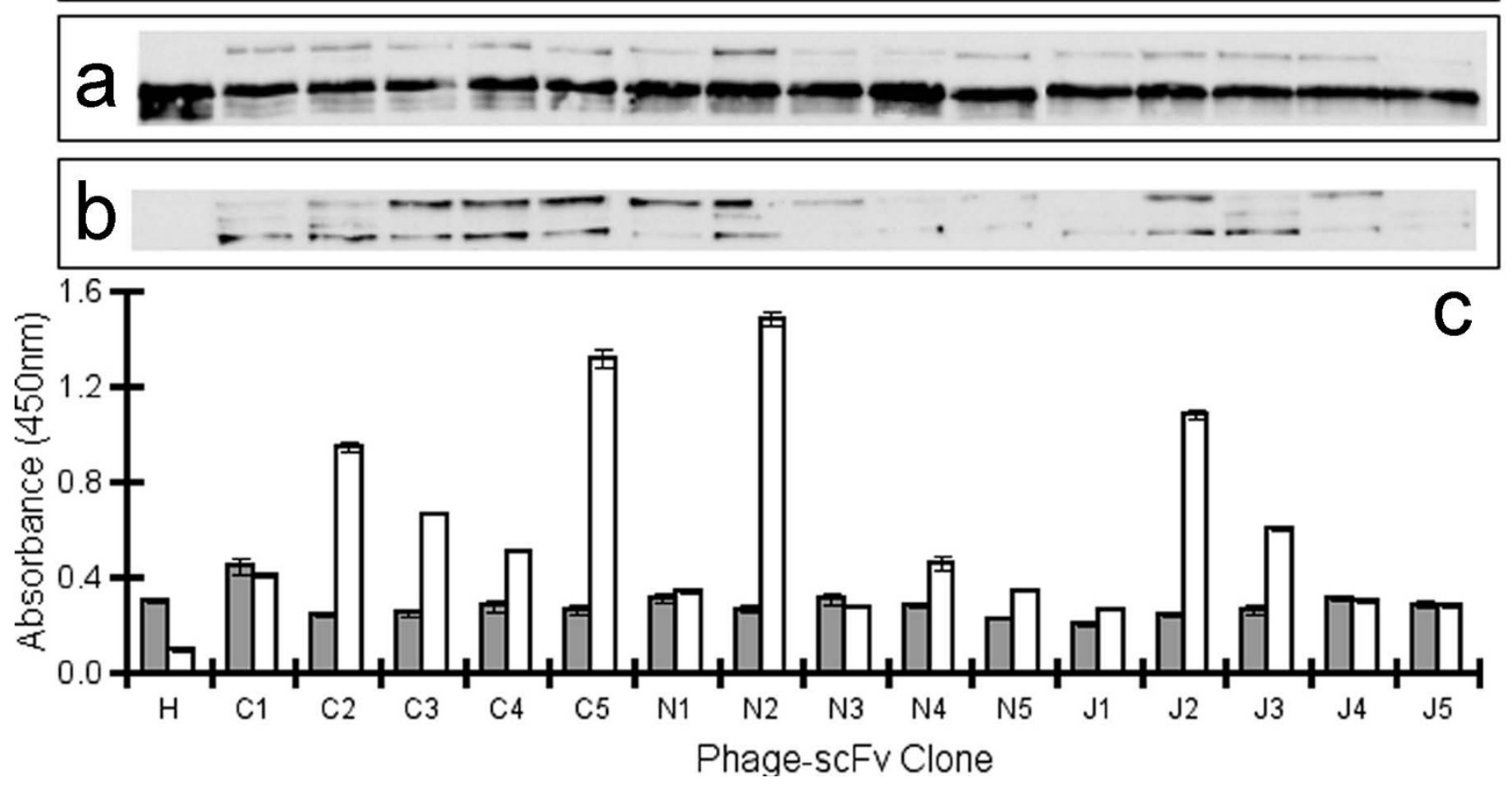

Figure I

Analysis of scFv phage display propensity. Each of the I 5 scFv-phage clones and helper phage were produced in $E$. coli (XLI-Blue), equalised, electrophoresed by SDS-PAGE, blotted and probed with (a) anti-plll antibody to demonstrate equalised phage titre or (b) anti-his ${ }_{6}$ antibody to detect varying levels in display of scFv on phage, as described above. The absolute chemiluminescent signals were measured by densitometry. (c) Binding to undiluted phage coated onto an immunosorbent plate was measured by ELISA to determine levels of plll and his ${ }_{6}$ using the same antibodies above. ELISA error bars represent standard error from triplicate measurements. Grey bars represent mean anti-plll signal and white are mean anti-his signal. A representative data set is shown from one batch of each phage-scFv for both the western blot and ELISA methods. Three independently produced batches of phage were analysed.

weight products, the result of proteolytic degradation. The 15 anti-Hc phage fusions showed a dominant pIII signal at $70 \mathrm{kDa}$, that was consistent from clone to clone indicating uniform loading of phage particles displaying each of the anti-Hc scFvs and a less dominant higher MW band migrating at $100 \mathrm{kDa}$, the density of which varied from

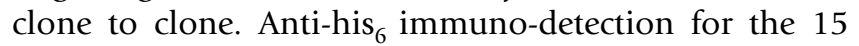
anti-Hc scFvs varied in intensity from clone to clone and was often observed in two bands at both $70 \mathrm{kDa}$ and 100 $\mathrm{kDa}$. Based on the distribution and signal of pIII and his ${ }_{6}$ bands it is possible to conclude that the $100 \mathrm{kDa}$ bands in Figure 1a solely represent full-length $\mathrm{scFv}$ fused to full length pIII. The $70 \mathrm{kDa}$ bands are likely to be due to both wild-type pIII (major species) and PIII appended to the his $_{6}$ tag of the scFv only (minor and indistinguishable). The remainder of the $\mathrm{scFv}$ molecule may have been cleaved away by proteolytic activity. It may also be possible that the $70 \mathrm{kDa}$ species represents full length $\mathrm{scFv}$ fused to degraded pIII (also indistinguishable). It is also worth noting that previous investigations reveal unexpected electrophoretic migration of pIII due to its high glycine content, extended shape and multi-domain structure leading to differences between calculated and expected molecular weights and this may have contributed to the results observed here $[33,34]$.

Quantitative laser scanning densitometry was used to measure the intensity of the chemiluminescent signal produced by the western blot analyses (data not shown). The total signal obtained from both the $100 \mathrm{kDa}$ and $70 \mathrm{kDa}$ anti-his $_{6}$ signals was used as a measure of display. Regardless of whether the full length scFv is present, detection of the his ${ }_{6}$ tag logically dictates display of that $\mathrm{scFv}$ protein on phage. This takes into account degradation (unlike the pIII blot signal). Data analysis was conducted using the AIDA Image Analyser Software and the absolute intensities were corrected for local background signal and normalised for phage loading (using anti-pIII signal) and 
then used to produce relative rankings of scFv display propensity for three independently produced batches of phage (See additional file 1). An ELISA platform was used to support the display level data obtained by western blot (Figure 1c). Coating of neat, PEG-purified phage (to remove any contaminating soluble scFv) without prior OD normalisation was amenable with this assay platform since variation in titre, as indicated by differences in pIII signal, could be accounted for by obtaining a ratio of his ${ }_{6}$ to $\mathrm{pIII} \mathrm{OD}_{450}$ values and this was used to produce display rankings.

Three independently-produced batches of the 15 anti-Hc phage-scFvs were analysed by both the western blot and ELISA method described above. Average rankings from 1 (highest displaying $\mathrm{scFv}$ ) to 15 (lowest displaying scFv) (Table 1) were used to conduct a Spearmans correlation analysis comparing rankings obtained between phage$\mathrm{scFv}$ batches and between the two measurement techniques. Ranks correlated well between batches and techniques (See additional file 1) with clones showing strong anti-his $_{6}$ western blot signals also showing high antihis $_{6}$ :anti-pIII ELISA ratios (e.g clones C3, C4, C5, N2 \& J2). Thus display level appears to be a non-batch dependent but clone-specific characteristic and varies markedly between clones in a panel of 15 anti-Hc scFvs.

This variability in display level was not unexpected given that these clones were derived after only 2 rounds of selec- tion on the Hc antigen (Scott et al, manuscript in preparation). Such differences in display level may have implications in selections such that scFvs exhibiting a higher display level may be preferentially enriched in the absence of vastly differing affinities. Given that scFvs have been shown to be relatively resistant to premature cytosolic folding/aggregation [31,32], we hypothesised that the display level variation shown here is due to variation in the overall expression level of the scFvs with the reasonable assumption that facility for translocation to the periplasm is uniform amongst the 15 clones.

\section{Overall scFv expression does not correlate with soluble levels in the periplasm and culture supernatant}

We found that the overall level of scFv expression defined as levels measured by western blot in the spheroplast fraction of E. coli HB2151 does not correlate with soluble levels in the periplasmic or culture supernatant fractions after $16 \mathrm{hr}$ culture induction.

Spheroplast, periplasmic and supernatant fractions were isolated from the cultures at the end of the $16 \mathrm{hr}$ induction period following normalisation of the culture density of each clone. Fractionated samples were electrophoresed and transferred onto nitrocellulose for probing with an anti-his $_{6}$ antibody (Figure 2a-c). The supernatant and periplasmic fractions of each anti-Hc scFv exhibited a single band at approximately $30 \mathrm{kDa}$ indicating fully processed scFv. The spheroplast fractions of most scFvs

Table I: Average relative rankings of scFv expression levels in various E. coli compartments and levels of display on filamentous phage.

\begin{tabular}{|c|c|c|c|c|c|c|}
\hline \multirow[b]{2}{*}{ Clone } & \multicolumn{4}{|c|}{ Average scFv Rankings } & \multicolumn{2}{|c|}{ Major Germline Families } \\
\hline & Supernatant & Periplasm & Spheroplast & Display & $\mathrm{VH}$ & VL \\
\hline $\mathrm{Cl}$ & $6.0 \pm 1$ & $9.8 \pm 0.8$ & $7.0 \pm 1.2$ & $11.7 \pm 1.1$ & $\mathrm{VH} 4$ & VL3 \\
\hline $\mathrm{C} 2$ & $6.0 \pm 0$ & $14.3 \pm 0.7$ & $3.0 \pm 0.6$ & $6.2 \pm 0.5$ & VHI & VL3 \\
\hline $\mathrm{C} 3$ & $7.5 \pm 0.5$ & $3.5 \pm 0.5$ & $8.7 \pm 2.9$ & $6.3 \pm 1.0$ & $\mathrm{VH} 4$ & VKI \\
\hline $\mathrm{C} 4$ & $14.5 \pm 0$ & $11.8 \pm 1.2$ & $4.0 \pm 1.5$ & $4.2 \pm 1.2$ & $\mathrm{VH} 4$ & VKI \\
\hline $\mathrm{C5}$ & $14.5 \pm 0$ & $6.0 \pm 1$ & $3.7 \pm 1.5$ & $2.7 \pm 0.3$ & $\mathrm{VH} 4$ & VKI \\
\hline NI & $10.0 \pm 2$ & $13.8 \pm 3.5$ & $10.0 \pm 0.6$ & $7.5 \pm 2.2$ & $\mathrm{VH} 2$ & VKI \\
\hline N2 & $4.0 \pm 0$ & $4.5 \pm 2.2$ & $3.3 \pm 1.9$ & $2.8 \pm 1.1$ & $\mathrm{VHI}$ & VKI \\
\hline N3 & $13.0 \pm 0$ & $10.8 \pm 0.5$ & $12.0 \pm 0$ & $11.5 \pm 0.9$ & $\mathrm{VH} 3$ & VKI \\
\hline N4 & $9.0 \pm 0$ & $3.5 \pm 0.5$ & $9.3 \pm 2.0$ & $12.2 \pm 1.1$ & $\mathrm{VH} 3$ & VL2 \\
\hline N5 & $8.3 \pm 3.3$ & $4.3 \pm 2.7$ & $11.0 \pm 1.0$ & $10.7 \pm 1.6$ & $\mathrm{VH} 3$ & VLI \\
\hline JI & $10.5 \pm 0.5$ & $9.0 \pm 3$ & $14.7 \pm 0.3$ & $12.2 \pm 1.1$ & VH3 & VL2 \\
\hline $\mathrm{j} 2$ & $2.0 \pm 0$ & $11.8 \pm 1.8$ & $4.0 \pm 1.5$ & $3.8 \pm 0.5$ & $\mathrm{VHI}$ & VKI \\
\hline $\mathrm{j} 3$ & $2.0 \pm 1$ & $3.8 \pm 2.2$ & $5.3 \pm 1.3$ & $6.5 \pm 1.3$ & VH3 & VL2 \\
\hline j4 & $2.0 \pm 1$ & $3.5 \pm 1.5$ & $10.0 \pm 1.0$ & $11.3 \pm 0.7$ & VH6 & VL2 \\
\hline $\mathrm{J} 5$ & $10.8 \pm 0.7$ & $10.0 \pm 1$ & $14.0 \pm 0.6$ & $11.3 \pm 1.5$ & $\mathrm{VH} 3$ & VL2 \\
\hline
\end{tabular}

Densitometry data derived from the western blots shown in Figures I and 2 were used to rank scFvs from I to I5 in order of highest to lowest levels of scFv respectively. Rankings from repeat experiments were averaged and are shown here along with standard error for the overall expression and display levels. The average relative spheroplast scFv level rankings were used as an indicator of overall expression level and to designate the scFvs into three groups of either "high (average ranking $\leq 5$; bold numbers)", "medium (average ranking $\geq 5$ but $\leq 10$; italicised numbers) " or "low" (average ranking $\geq \mathrm{II}$; No Shading) expression. Rankings for phage display propensity were also used to assign similar groupings. The major $\mathrm{V}_{\mathrm{H}}$ and $\mathrm{V}_{\mathrm{L}}$ germline designations for each scFv are also shown for each clone. 

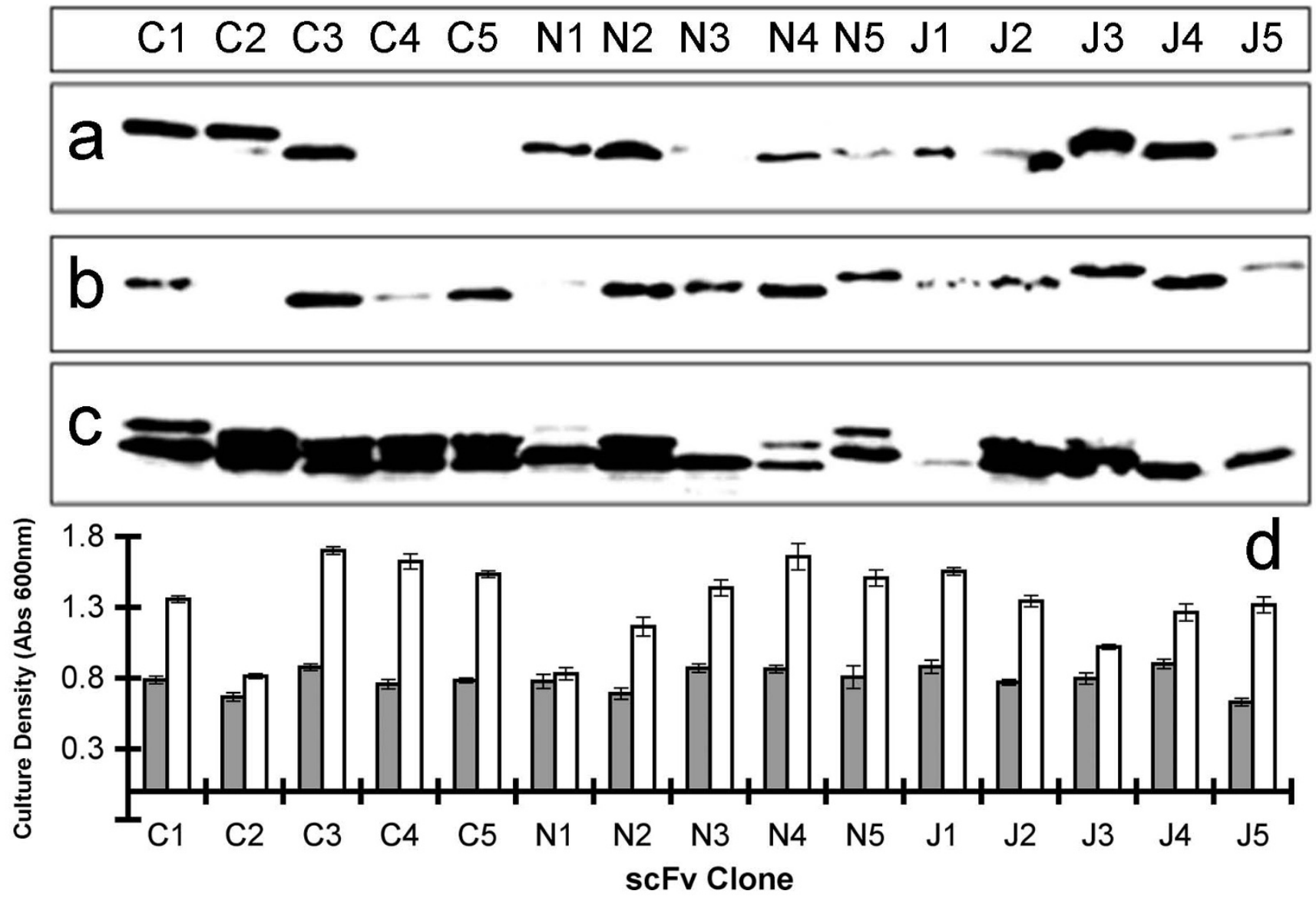

Figure 2

Compartmental analysis of overall relative levels of scFv expression in Escherichia coli. Each of the $15 \mathrm{scFv}$ clones were produced in $E$. coli $(\mathrm{HB} 2 \mathrm{I} 5 \mathrm{I})$. The cultures were equalised for bacterial density and the supernatant, periplasmic and spheroplast fractions were electrophoresed by SDS-PAGE and blotted. Anti-his 6 antibody was used to probe for the scFvs, developed by chemiluminescence. (a) supernatant fraction, (b) periplasmic fraction and (c) spheroplast $E$. coli pellet. The blot is representative of three independent experiments conducted on independently produced samples. (d) Pre-induction (grey bars) and final culture densities (white bars) measured by $\mathrm{OD}_{600}$ are also shown and represent mean values from three independent experiments.

contained doublet bands at approximately $30 \mathrm{kDa}$, likely to represent both $\mathrm{N}$-terminally processed and unprocessed $\mathrm{scFv}$ in addition to smaller breakdown products. The absolute signals vary markedly between scFv clones in each of the three fractions. Quantitative laser scanning densitometry measurements of western blot chemiluminescence were acquired (data not shown) and used to rank scFvs from 1 (high) to 15 (low) in order of densitometry signals for levels of $\mathrm{scFv}$ present in the E. coli spheroplast, periplasmic lysate and culture supernatant. The overall rankings were reproducible between data sets (See additional file 1) for the same E. coli compartment indicating that overall scFv expression (spheroplast level) and localisation to the periplasm and culture supernatant in soluble form are clone-specific characteristics, that vary from clone to clone.
Average rankings were produced for each compartment using independently produced data sets (Table 1) and these were used to conduct a Spearmans correlation test in order to determine if relative levels of $\mathrm{scFv}$ present within the three fractions correlate with each other for each clone. For $\mathrm{n}=15$, a Rho value greater than 0.441 indicates positive correlation with a p-value of less than or equal to 0.05 . However, comparison of supernatant scFv levels with periplasmic or spheroplast levels yielded coefficients of 0.38 and 0.37 respectively. Comparison of periplasmic and spheroplast levels yielded a coefficient of 0.13 . These data suggest there is no significant correlation ( $p>0.05)$ between the overall expression level of a scFv molecule as defined by its spheroplast level, and its relative soluble levels within the periplasmic space or culture supernatant. 
Interestingly in three independent experiments the final culture density, as determined by $\mathrm{OD}_{600}$ after a $16 \mathrm{hr}$ induction, reached a value reproducibly characteristic to the clone being studied ranging from 0.8 for $\mathrm{C} 2$ to 1.7 for N4 (Figure 2d). Relative increases in culture density following induction were calculated using the mean culture density data shown in Figure $2 \mathrm{~d}$. The relative increases were used to rank the scFvs from 1 (highest fold increase) to 15 (lowest fold increase) and used to determine Spearmans correlation coefficients in pair-wise comparisons with spheroplast, periplasmic and culture supernatant scFv level rankings. Although there was no detectable correlation between relative increase in culture density ranking and spheroplast or periplasmic scFv level rankings, there was a moderate but statistically significant negative correlation $($ Rho $=-0.55, \mathrm{p}<0.05)$ when compared with culture supernatant $\mathrm{scFv}$ levels. Thus $\mathrm{scFv}$ clones that exhibit a low relative level of culture supernatant localisation allow the bacteria harbouring them to attain a higher culture density than other scFvs.

Given that scFvs are not prone to cytosolic aggregation, the lack of correlation between the spheroplast and periplasmic levels of scFv may be explained by differential toxicity once in the periplasm. Our culture density data suggests that some scFvs are more toxic than others and that clones with a high level of supernatant localisation hinder bacterial growth following induction, perhaps through lytic mechanisms or destabilisation of the outer membrane. This non-uniform differential soluble scFv localisation therefore directly influences any correlation between supernatant levels and periplasm, and supernatant/periplasm with spheroplast levels. Differing levels of toxicity may be due to periplasmic aggregation and hence inclusion body formation, proteolytic breakdown or misfolding due to inappropriate disulphide bond formation which has been observed previously for scFvs $[23,35]$.

\section{Phage display propensity exhibits strong positive correlation with overall scFv expression Levels}

The average relative $\mathrm{scFv}$ rankings for display level propensity were compared with the expression analysis of non-fused scFv. We found that display level ranking does not correlate with soluble scFv level rankings in either the periplasm $(\mathrm{Rho}=0.25 ; \mathrm{p}>0.05)$ or supernatant $(\mathrm{Rho}=$ $0.20 ; \mathrm{p}>0.05)$. However, we found a strong positive correlation $($ Rho $=0.88 ; \mathrm{p}<0.005)$ between display level ranking and overall scFv expression ranking as defined by relative spheroplastic levels, illustrated in Figure 3. Based on the ranking data, scFv clones were grouped into 'high', 'medium' or 'low' for phage display propensity and overall expression levels (Table 1). Display ranking was achieved using two independent methods on three independent batches of material and was remarkable consistent (See additional file 1). The average ranking was at

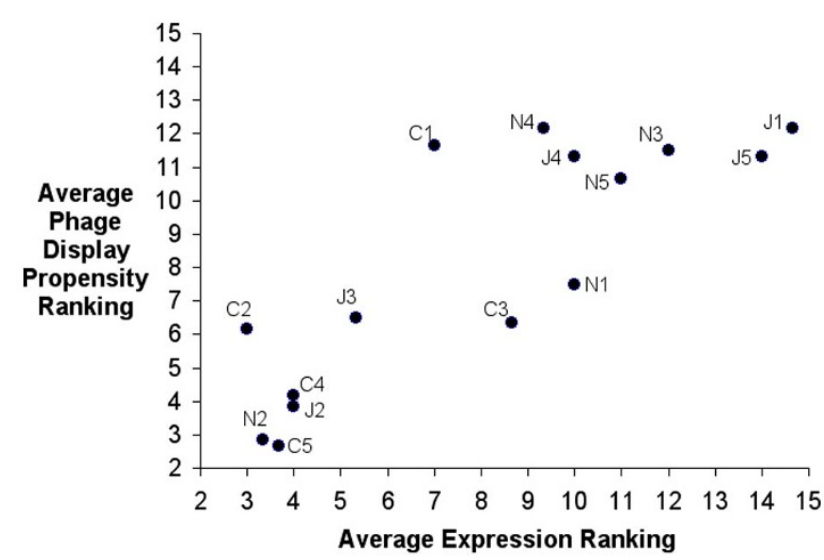

Figure 3

Overall levels of scFv expression exhibit strong positive correlation with phage display propensity. Average relative rankings obtained for overall scFv expression in the spheroplast fraction of $E$. coli are displayed on a scatterplot in relation to average relative phage display propensity rankings for each individual clone. A ranking of I represents a high relative level of overall expression or phage display propensity and a ranking of 15 represent a low relative level of overall expression or phage display propensity. The plot shows a strong positive correlation between expression ranking and display level ranking. Each $\mathrm{scFv} x$-axis co-ordinate represents the average ranking from 3 independent experimental observations of overall scFv expression (Table I). Each scFv y-axis co-ordinate represents the average ranking from 6 independent experimental observations of display level (three ELISA and three western blots) carried out on three batches of phage-scFv (Table I). The Spearmans coefficient of rank correlation Rho coefficient for this association is 0.88 representing strong positive correlation with a $p$-value $<0.005$. Standard errors are not conventionally used for averages of ranks. However they have been calculated (Table I) and are not shown graphically for purposes of clarity.

worst, only varied by 2 places (clone N1). Total expression ranking was also very consistent with clone $\mathrm{C} 3$ being the most variable. Taking all factors into account, we believe this to be a robust and strong correlation.

This interesting correlation provides insight into the possible mechanism leading to a lack of correlation between overall scFv expression and soluble levels in the periplasm and supernatant. Assembly of pIII-scFv fusion proteins into phage particles is dependant upon their translocation to the periplasm [30]. Since overall expression level correlates strongly with display level, it may be concluded that few of these scFv clones exhibit a marked level of premature folding or degradation in the bacterial cytosol that may hinder their translocation to the periplasm. Any degree of premature folding may be assumed to be uniform amongst the 15 clones since the only factor influenc- 
ing display level is simply the absolute level of scFv expressed in the bacterial cytosol. Given that scFv expression in both pIII-fused and non-fused forms share the same secretary signal sequence it can be assumed that both are trafficked to the periplasm in an identical manner. We would therefore expect levels of soluble scFv in the periplasm to correlate with overall expression level and display level. This was not the case.

A hypothetical explanation for the observed disparity therefore potentially lies in the fact that the fates of pIII fused and non-fused scFvs diverge once the proteins are translocated across the inner membrane of $E$. coli. The pIII-scFv fusion remains anchored in the inner membrane via the C-terminal domain of pIII [13] whereas free scFv remains in the periplasmic space where it is subject to potential mis-folding or aggregation. We propose that differential levels of aggregation between clones at this stage leads to varying degrees of membrane destabilisation and therefore leaking of $\mathrm{scFv}$ into the supernatant.

\section{Codon usage indirectly influences scFv phage display propensity}

Figure 4 shows the amino acid sequences of the 15 antitetanus toxin Hc scFvs aligned by homology. The major germline constituents of the $s c F v V_{H}$ and $V_{L}$ domains were determined (Table 1) in order to evaluate whether particular germline sequences are more amenable to expression in E. coli and hence display on phage. It seems that scFvs exhibiting a high level of display and expression often harbour a VK1 derived $V_{L}$ domain and those exhibiting low expression and display tend to harbour a $\mathrm{VH} 3$ derived $\mathrm{V}_{\mathrm{H}}$ domain. These observations support earlier work where Ewert et al [31] analysed total soluble expression of individual and paired $\mathrm{V}$-domains. Here also, VH3 subtype was best and most soluble $\mathrm{VH}$ domain and VK1 was also ranked highly. It was not possible to elucidate definite D and $\mathrm{J}$ segment germline designations for the $\mathrm{scFv} \mathrm{V}_{\mathrm{H}}$ and $\mathrm{V}_{\mathrm{L}}$ domains since they appear to have undergone significant affinity maturation. This is not unexpected as the library used was derived from a pool of individuals many of whom were likely to have been immunised against tetanus, leading to antibody affinity maturation.

The link between total protein expression and phage display is supported by bioinformatics analyses. A number of factors influence translation efficiency, codon usage being an important parameter $[14,15]$. One codon usage index is the codon adaptation index (CAI) [36] which was determined using the codonW programme [37]. Ranking of the CAI correlates with overall expression and hence phage display $(\mathrm{Rho}=0.68 \mathrm{p}<0.01)$. At the nucleotide level, the scFv sequences are similar (See additional file 2) and the CAI covers a small range, suggesting that minor differences in codons could account for large differences in dis-

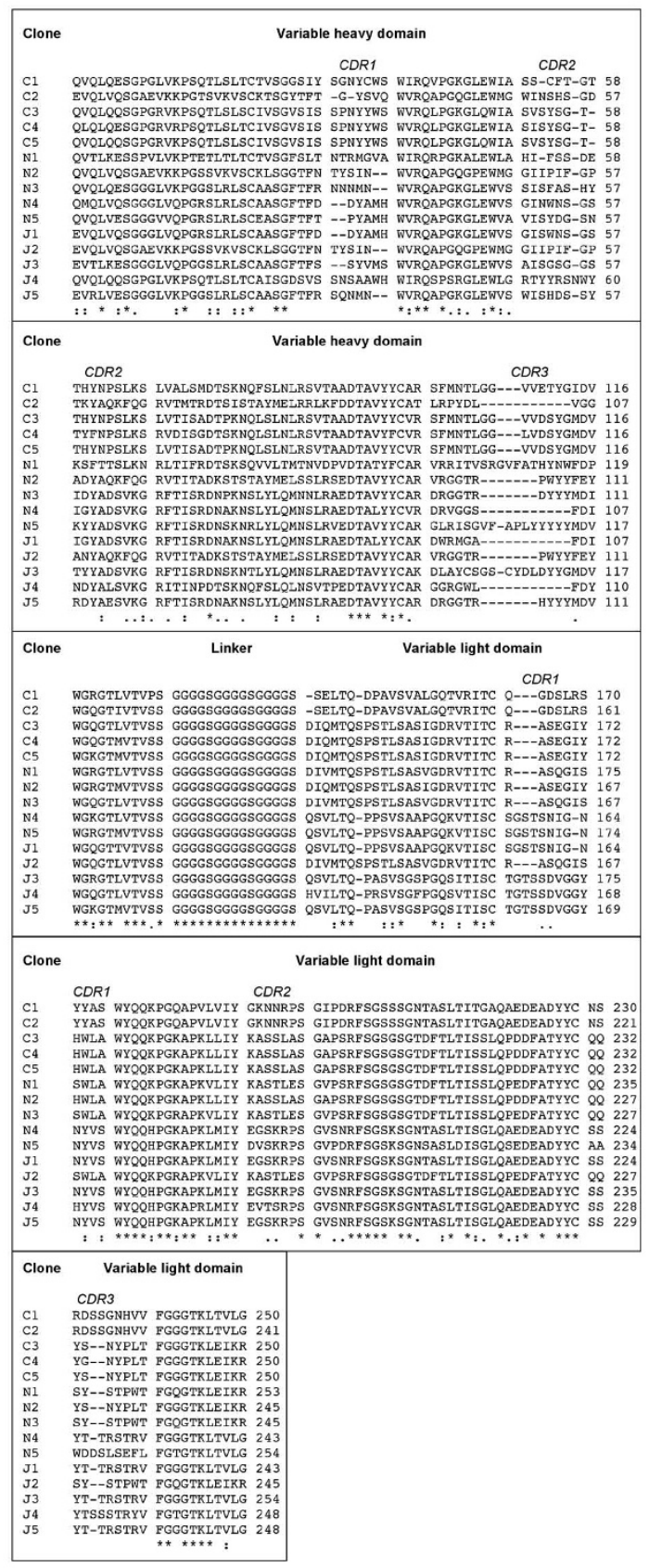

Figure 4

Annotated amino acid sequence alignment of the 15 anti-Hc scFvs. The amino acid sequences of 15 anti-Hc scFvs $\mathrm{Cl}-\mathrm{C} 5, \mathrm{JI}-\mathrm{J} 5$ and $\mathrm{NI}-\mathrm{N} 5$ were aligned using ClustalW [45]. The inter-domain linker $\left(\mathrm{G}_{4} S\right)_{3}$ joins the $\mathrm{N}$-terminal $\mathrm{V}_{\mathrm{H}}$ domain to the $\mathrm{C}$-terminal $\mathrm{V}_{\mathrm{L}}$ domain of the scFv. Within each of the $\mathrm{V}_{\mathrm{H}}$ and $\mathrm{V}_{\mathrm{L}}$ domains, there are three areas of increased diversity indicating the hypervariable complimentarity determining regions (labelled). Identically-conserved residues are labelled $(*)$, conserved residues are labelled $(:)$ and similar residues are labelled (.) The protein and corresponding DNA sequences (See additional file 2) have been deposited in the EMBL database. 
play and expression. The Improbizer program [38] was used to search for DNA motifs which could be linked to high expression. However, many motifs were identified and given the similar nucleotide backgrounds, it was impossible to deduce which ones could be of significance and which ones were signatures of the V-gene sequences. A similar protein sequence analysis failed to identify any protein motifs which correlate with phage display propensity. This confirms that phage display of scFvs is closely and significantly linked to overall protein expression and not post-translational protein-orientated events such as folding. There is a great deal of research detailing protein sequence motifs which affect functional protein expression, particularly recombinant antibodies $[23,39]$. This is likely to affect the levels of scFv found in the supernatant. Antibody V-domains are generally homologous with a consensus homology of approximately 50\% [40]. The data in this study comprises $\mathrm{V}$-domains ranging from highly similar sequences to around 60\% homology (Fig. $4)$. Given that even a single residue change can influence major biophysical properties [23], making observations with highly similar sequences is still valid for this comparative analysis.

\section{Conclusion}

In conclusion, when premature protein folding in the bacterial cytosol is not a factor, phage display levels correlate with overall expression level. Higher levels of synthesised protein result in greater levels of inner membrane translocation and incorporation into phage particles. Therefore, factors influencing overall expression indirectly influence phage display propensity. This suggests that better $\mathrm{scFv}$ display could be achieved in codon-optimised strains of E. coli such as those commercially available or in vectors with very strong promoters such as the T7-promoter system. However, high levels of overall expression may not necessarily imply high levels of soluble localisation to the periplasm or supernatant. This may influence selection of clones for further study after phage display suggesting that a parallel screen of periplasmic or supernatant levels should be conducted in order to select clones that exhibit a desirable high soluble yield in addition to phage ELISAs. The work also shows how scFv display level and expression can vary considerably amongst clones despite an overall high level of sequence homology.

\section{Materials and methods}

\section{Production and isolation of phage-scFv fusions}

A description of the selection and full characterisation of these 15 anti-tetanus toxin scFvs is in preparation (Scott, $\mathrm{N}$ et al, manuscript in preparation). All scFvs were selected on plastic immobilised recombinant tetanus toxin Hcdomain using Nunc immunotubes at high antigen concentrations. This allowed selection of a diverse array of binders with no selection pressure on affinity, display or expression.
The amber suppressor XL1-Blue strain of Escherichia coli (Stratagene) was transformed [41] with the phagemid vector pCANTAB6 [29] encoding each of the fifteen anti-Hc scFvs C1-C5, N1-N5 and J1-J5 under the control of the lac promoter. Individual transformant colonies of each scFv clone were selected and grown for 16 hours in a $5 \mathrm{ml}$ culture of 2TY [41] containing $100 \mu \mathrm{g} / \mathrm{ml}$ carbenicillin, $15 \mu \mathrm{g} /$ $\mathrm{ml}$ tetracycline and $1 \%$ glucose, with shaking at $250 \mathrm{rpm}$. The cultures were sub-cultured, after removal of old medium, at a 1:100 ratio into $50 \mathrm{ml}$ of fresh medium and grown at $37^{\circ} \mathrm{C}$ with shaking at $250 \mathrm{rpm}$. At an $\mathrm{OD}_{600}$ of $0.4-0.5$, the phagemid containing bacteria were infected with VCSM13 helper phage (Stratagene) at a multiplicity of infection of 20:1 [42]. Infection was allowed to take place for 30 minutes at $37^{\circ} \mathrm{C}$ without shaking followed by a further 30 minutes at $37^{\circ} \mathrm{C}$ with shaking at $200 \mathrm{rpm}$. The bacteria were isolated by centrifugation and resuspended in 50 $\mathrm{ml}$ of $2 \mathrm{TY}$ containing $100 \mu \mathrm{g} / \mathrm{ml}$ carbenicillin and $50 \mu \mathrm{g} / \mathrm{ml}$ kanamycin. The cultures were incubated overnight at $30^{\circ} \mathrm{C}$ with shaking at $300 \mathrm{rpm}$. The culture supernatants were clarified by centrifugation and filtered through a $0.44 \mu \mathrm{m}$ membrane. A third volume of ice-cold $20 \%$ polyethylene glycol 8000 containing $2.5 \mathrm{M} \mathrm{NaCl}$ was mixed with the phage containing supernatant and the solution incubated for 2 hours at $4{ }^{\circ} \mathrm{C}$. Precipitated phage were isolated by centrifugation at $4000 \mathrm{rpm}$ for 1 hour at $4{ }^{\circ} \mathrm{C}$ and resuspended in commercial phosphate buffered saline (PBS) ( $\mathrm{pH}$ 7.4) to a volume one tenth the original culture volume. A final centrifuge step at 13,000 rpm was used to remove any debris. Phage titre was estimated for each phage-scFv clone using $\mathrm{UV}$ spectroscopy $\left(\mathrm{OD}_{270}=1\right.$ is equivalent to $1.1 \times 10^{13}$ phage particles per millilitre) [43].

\section{Expression of non-fused scFv and isolation of spheroplast, periplasmic and supernatant fractions}

The DNA encoding the fifteen anti-Hc scFvs in phagemid pCANTAB6 were transformed into the HB2151 (GE Healthcare, UK) strain of $E$. coli which produces only scFv rather than a mixture of non-fused $\mathrm{scFv}$ and scFv-pIII fusion since translation terminates at the amber TAG codon separating the two genes. The clones were grown for 16 hours at $37^{\circ} \mathrm{C}$ in $2 \mathrm{TY}$ broth containing $1 \%$ glucose and $100 \mu \mathrm{g} / \mathrm{ml}$ carbenicillin with shaking at $250 \mathrm{rpm}$. After removal of old medium the cells were sub-cultured at a 1:100 ratio into $10 \mathrm{ml}$ of fresh medium and grown to an $\mathrm{OD}_{600}$ of between 0.6 and 0.8 at $37^{\circ} \mathrm{C}$ with shaking at $250 \mathrm{rpm}$ [42]. At this point the bacteria were isolated by centrifugation and resuspended in $10 \mathrm{ml}$ of 2TY medium containing $100 \mu \mathrm{g} / \mathrm{ml}$ carbenicillin and $1 \mathrm{mM}$ IPTG to induce expression of the non-fused $\mathrm{scFv}$ genes. The cultures were incubated overnight at $30^{\circ} \mathrm{C}$ with shaking at $300 \mathrm{rpm}$. The $\mathrm{OD}_{600}$ was measured at the end of this period and the culture volumes adjusted with 2TY broth in order to normalise the bacterial density to that of the lowest clone. The cultures were centrifuged at $4000 \mathrm{rpm}$ for 1 hour at $4^{\circ} \mathrm{C}$ and the supernatant retained. The bac- 
terial pellets were re-suspended in ice-cold periplasmic extraction buffer containing $500 \mathrm{mM}$ sucrose $100 \mathrm{mM}$ Tris-HCl, 1 mM EDTA pH 8.0 [44]. The volume of buffer used was one-tenth the final OD normalised volume. The resuspended bacteria were mixed by vortexing for $10 \mathrm{sec}-$ onds every 5 minutes for 20 minutes in order to break open the outer membrane of the bacteria. Spheroplasts were then isolated by centrifugation at $13,000 \mathrm{rpm}$ for 30 minutes at $4{ }^{\circ} \mathrm{C}$ and the supernatant (periplasmic fraction) retained. The spheroplast containing pellet was resuspended in distilled $\mathrm{H}_{2} \mathrm{O}$ at one-tenth the volume of OD normalised culture volume.

\section{Measurement of phage display levels and non-fused scFv expression using western blot with quantitative laser scanning densitometry analysis}

For measurement of phage display propensity, equal numbers of phage displaying each of the $15 \mathrm{scFv}$ clones and a helper phage control were subject to reducing SDS-PAGE and subsequently electroblotted onto nitrocellulose. Each clone and the helper phage were probed with a murine anti-pIII antibody (Clone 10C3, MoBiTec, Germany-which recognises a 10 residue C-terminal epitope, thus suitable for investigating degraded scFv-pIII fusion proteins) followed by a goat anti-mouse HRPO secondary. Simultaneously the samples were probed with HRPO conjugated murine anti-his ${ }_{6}$ antibody (Sigma). The detection of pIII is a measure of total phage present and the measurement of the his ${ }_{6}$ tag indicates levels of scFv displayed. In the case of non-fused scFv, the supernatant $(5 \mu \mathrm{l})$, periplasmic $(0.5 \mu \mathrm{l})$ and supernatant $(0.5 \mu \mathrm{l})$ fractions were subjected to reducing SDS-PAGE and electroblotted onto nitrocellulose. Probing with anti-his ${ }_{6} \mathrm{HRPO}$ was used to measure the scFv levels. In both the case of measuring levels of scFv fused to phage and of non-fused scFv, western blots were developed using the ECL plus western blotting detection system. Chemiluminescent signals were detected and measured using quantitative laser scanning densitometry [LAS3000 CCD Imaging System] (Fujifilm, Japan) facilitated with the AIDA Biopackage image analyser software (Raytest, Germany). Absolute signal intensities derived from the chemiluminescent signal were corrected for local background signal and used to produce a relative ranking of scFv display level and levels of non-fused scFv in spheroplast, periplasm and supernatant.

\section{Measurement of scFv display level by ELISA}

As an additional measurement of scFv levels on phage, an ELISA methodology was used. Undiluted phage $(100 \mu \mathrm{l})$ were coated onto 96-well Maxisorp Nunc-Immunoplates. Following a blocking step with Marvel ${ }^{\mathrm{TM}}$ dried milk powder PIII and his ${ }_{6}$ immunodetection were facilitated with the antibodies used during the western blot procedure. The assay was developed using BM-Blue POD substrate and stopped with $1 \mathrm{M}$ HCL. Binding signal $\left(\mathrm{OD}_{450}\right)$ was measured using a SpectraMax 340PC plate reader. A ratio of pIII to his ${ }_{6} \mathrm{OD}_{450}$ values was used as an indication of relative display level to account for variation in phage titre and rankings were produced accordingly.

\section{Statistical analyses}

Statistical assessment including derivation of means and standard errors was carried out using the Sigma Plot Software Version 8.0 (Systat Software Inc., London). Comparison of scFv rankings between different sample sets was facilitated through calculation of the Spearmans Coefficient of Rank Correlation parameter (Rho) which shows the degree of correlation and its statistical significance.

\section{Abbreviations}

$\mathrm{OD}_{600}$ : Optical density at $600 \mathrm{~nm}$; HRPO: Horse-radishperoxidise; ELISA: Enzyme linked immuno-sorbant assay; scFv: Single chain Fv; E. coli: Escherichia coli; $\mathrm{H}_{\mathrm{c}}$ : Tetanus toxin heavy chain $\mathrm{C}$-terminal domain; his 6 : Hexahistidine epitope tag; pIII: Minor protein 3 of phage.

\section{Authors' contributions}

OQ and MW originally selected the anti-toxin scFvs under the direction of NF and MD. NS carried out the scFv-phage identifications, preparations, scFv expression, display analyses, statistical analyses under the direction of MD. NS and MD drafted the manuscript. CR carried out some of the scFv-phage preparations and display analyses. All authors read, contributed to and approved the final manuscript.

\section{Additional material}

\section{Additional file 1}

Individual rankings from the independent batches of scFv-phage in the different experiments. This shows the consistency and reliability of the data used to derive the correlations.

Click here for file

[http://www.biomedcentral.com/content/supplementary/14726750-8-97-S1.doc]

\section{Additional file 2}

Annotated nucleotide sequence alignment of the 15 anti-Hc scFvs. The DNA sequences of fifteen anti-Hc scFus C1-C5, J1-J5 and N1-N5 were aligned using ClustalW [45]. Conserved bases are labelled $\left({ }^{*}\right)$.

Click here for file

[http://www.biomedcentral.com/content/supplementary/14726750-8-97-S2.tiff]

\section{Acknowledgements}

NS and CR are funded by the Wellcome Trust 4-year PhD programme in the Molecular and Cellular Basis of Infection. MW was supported by a grant from the Leventis Foundation. OQ was funded by a BBSRC studentship and a project grant to NF. We thank Dr. Darek Kedra (Imperial College Centre for Bioinformatics) for help with bioinformatics analyses and MedlmmuneAstraZenneca for use of their single-chain Fv phage library. 


\section{References}

I. Holliger P, Hudson PJ: Engineered antibody fragments and the rise of single domains. Nat Biotechnol 2005, 23: I I 26- I I 36.

2. Carter PJ: Potent antibody therapeutics by design. Nat Rev Immunol 2006, 6:343-357.

3. Filpula D: Antibody engineering and modification technologies. Biomol Eng 2007, 24:201-215.

4. Chang H, Qin W, Li Y, Zhang J, Lin Z, Lv M, Sun Y, Feng J, Shen B: A novel human scFv fragment against TNF-alpha from de novo design method. Mol Immunol 2007, 44:3789-3796.

5. Luginbuhl B, Kanyo Z, Jones RM, Fletterick RJ, Prusiner SB, Cohen FE, Williamson RA, Burton DR, Pluckthun A: Directed evolution of an anti-prion protein scFv fragment to an affinity of I pM and its structural interpretation. I Mol Biol 2006, 363:75-97.

6. Kubetzko S, Balic E, Waibel R, Zangemeister-Wittke U, Pluckthun A: PEGylation and multimerization of the anti-p I 85HER-2 single chain Fv fragment 4D5: effects on tumor targeting. J Biol Chem 2006, 28I:35186-3520I.

7. Fabbrini M, Trachsel E, Soldani P, Bindi S, Alessi P, Bracci L, Kosmehl $H$, Zardi L, Neri D, Neri P: Selective occlusion of tumor blood vessels by targeted delivery of an antibody-photosensitizer conjugate. Int J Cancer 2006, I I 8: |805-|8|3.

8. Schwemmlein M, Peipp M, Barbin K, Saul D, Stockmeyer B, Repp R, Birkmann J, Oduncu F, Emmerich B, Fey GH: A CD33-specific single-chain immunotoxin mediates potent apoptosis of cultured human myeloid leukaemia cells. Br J Haematol 2006, I33:|4|-I5I.

9. Bradbury AR, Marks JD: Antibodies from phage antibody libraries. J Immunol Methods 2004, 290:29-29.

10. Clackson T, Hoogenboom HR, Griffiths AD, Winter G: Making antibody fragments using phage display libraries. Nature 1991, 352:624-628.

II. Hoogenboom HR: Selecting and screening recombinant antibody libraries. Nat Biotechnol 2005, 23: I I05-I I I6.

12. McCafferty J, Griffiths AD, Winter G, Chiswell DJ: Phage antibodies: filamentous phage displaying antibody variable domains. Nature 1990, 348:552-554.

13. Marvin DA: Filamentous phage structure, infection and assembly. Curr Opin Struct Biol 1998, 8:150-158.

14. Lu P, Vogel C, Wang R, Yao X, Marcotte EM: Absolute protein expression profiling estimates the relative contributions of transcriptional and translational regulation. Nat Biotechnol 2007, 25: I I 7-124.

15. Makrides SC: Strategies for achieving high-level expression of genes in Escherichia coli. Microbiol Rev 1996, 60:512-538.

16. Dyson MR, Shadbolt SP, Vincent KJ, Perera RL, McCafferty J: Production of soluble mammalian proteins in Escherichia coli: identification of protein features that correlate with successful expression. BMC Biotechnol 2004, 4:32-40.

17. Missiakas D, Schwager F, Betton JM, Georgopoulos C, Raina S: Identification and characterization of HsIV HsIU (CIpQ ClpY) proteins involved in overall proteolysis of misfolded proteins in Escherichia coli. EMBO J 1996, I 5:6899-6909.

18. Bowden GA, Paredes AM, Georgiou G: Structure and morphology of protein inclusion bodies in Escherichia coli. Biotechnology (NY) I991, 9:725-730.

19. Baneyx F, Mujacic M: Recombinant protein folding and misfolding in Escherichia coli. Nat Biotechnol 2004, 22:|399-|408.

20. Betton JM, Sassoon N, Hofnung M, Laurent M: Degradation versus aggregation of misfolded maltose-binding protein in the periplasm of Escherichia coli. J Biol Chem 1998, 273:8897-8902.

21. De Sutter K, Remaut E, Fiers W: Disulphide bridge formation in the periplasm of Escherichia coli: beta-lactamase:: human IgG3 hinge fusions as a model system. Mol Microbiol 1992, 6:2201-2208.

22. Forsberg G, Forsgren M, Jaki M, Norin M, Sterky C, Enhorning A, Larsson K, Ericsson M, Bjork P: Identification of framework residues in a secreted recombinant antibody fragment that control production level and localization in Escherichia coli. J Biol Chem 1997, 272:12430-12436.

23. Nieba L, Honegger A, Krebber C, Pluckthun A: Disrupting the hydrophobic patches at the antibody variable/constant domain interface: improved in vivo folding and physical characterization of an engineered scFv fragment. Protein Eng 1997 1 0:435-444
24. Huber D, Boyd D, Xia Y, Olma MH, Gerstein M, Beckwith J: Use of thioredoxin as a reporter to identify a subset of Escherichia coli signal sequences that promote signal recognition particle-dependent translocation. J Bacteriol 2005, I 87:2983-299I.

25. Baneyx F, Palumbo JL: Improving heterologous protein folding via molecular chaperone and foldase co-expression. Methods Mol Biol 2003, 205: I7|-197.

26. Bothmann H, Pluckthun A: Selection for a periplasmic factor improving phage display and functional periplasmic expression. Nat Biotechnol 1998, 16:376-380.

27. Kipriyanov SM, Moldenhauer G, Little M: High level production of soluble single chain antibodies in small-scale Escherichia coli cultures. J Immunol Methods 1997, 200:69-77.

28. Ulrich HD, Patten PA, Yang PL, Romesberg FE, Schultz PG: Expression studies of catalytic antibodies. Proc Natl Acad Sci USA 1995, 92:II907-II9|।.

29. Vaughan TJ, Williams AJ, Pritchard K, Osbourn JK, Pope AR, Earnshaw JC, McCafferty J, Hodits RA, Wilton J, Johnson KS: Human antibodies with sub-nanomolar affinities isolated from a large non-immunized phage display library. Nat Biotechnol 1996, 14:309-314.

30. Steiner D, Forrer P, Stumpp MT, Pluckthun A: Signal sequences directing cotranslational translocation expand the range of proteins amenable to phage display. Nat Biotechnol 2006, 24:823-831

31. Ewert S, Huber T, Honegger A, Pluckthun A: Biophysical properties of human antibody variable domains. J Mol Biol 2003, 325:531-553.

32. Thie H, Schirrmann T, Paschke M, Dübel S, Hust M: SRP and Sec pathway leader peptides for antibody phage display and antibody fragment production in E. coli. New Biotechnology 25:49-54.

33. Chatellier J, Hartley O, Griffiths AD, Fersht AR, Winter G, Riechmann L: Interdomain interactions within the gene 3 protein of filamentous phage. FEBS Lett 1999, 463:371-374.

34. Gailus V, Rasched I: The adsorption protein of bacteriophage fd and its neighbour minor coat protein build a structural entity. Eur J Biochem 1994, 222:927-931.

35. Kipriyanov SM, Moldenhauer G, Martin AC, Kupriyanova OA, Little $M$ : Two amino acid mutations in an anti-human CD3 single chain Fv antibody fragment that affect the yield on bacterial secretion but not the affinity. Protein Eng 1997, 1 0:445-453.

36. Sharp PM, Li WH: The codon Adaptation Index - a measure of directional synonymous codon usage bias, and its potential applications. Nucleic Acids Res | 987, | 5: | 28|- 295.

37. Sharp PM: Determinants of DNA sequence divergence between Escherichia coli and Salmonella typhimurium: codon usage, map position, and concerted evolution. I Mol Evol 1991, 33:23-33.

38. Ao W, Gaudet J, Kent WJ, Muttumu S, Mango SE: Environmentally induced foregut remodeling by PHA-4/FoxA and DAF-I 2 / NHR. Science 2004, 305: $1743-6$.

39. Bothmann H, Pluckthun A: The periplasmic Escherichia coli peptidylprolyl cis,trans-isomerase FkpA. I. Increased functional expression of antibody fragments with and without cis-prolines. I Biol Chem 2000, 275:17100-17105.

40. Tomlinson IM, Cook GP, Carter NP, Elaswarapu R, Smith S, Walter G, Buluwela L, Rabbitts TH, Winter G: Human immunoglobulin VH and D segments on chromosomes I5qII.2 and I6p II.2. Hum Mol Gen 1994, 3:853-860.

4I. Sambrook J, Russell DW: Molecular Cloning: A Laboratory Manual Cold Spring Harbor Laboratory Press, New York; 200 I.

42. Pope AR, Embleton MJ, Mernaugh R: Construction and use of antibody gene repertoires. In Antibody Engineering: A Practical Approach Edited by: McCafferty J, Hoogenboom HR, Chiswell DJ. Oxford University Press, London; 1996:23-27.

43. Russel M, Lowman HB, Clackson T: Introduction to phage biology and phage display. In Phage Display Edited by: Clackson T, Lowman B. Oxford University Press, London; 2004: 16.

44. Hust M, Jostock T, Menzel C, Voedisch B, Mohr A, Brenneis M, Kirsch MI, Meier D, Dubel S: Single chain Fab (scFab) fragment. BMC Biotechnol 2007, 7:14.

45. Thompson JD, Higgins DG, Gibson TJ: CLUSTAL W: improving the sensitivity of progressive multiple sequence alignment through sequence weighting, position-specific gap penalties and weight matrix choice. Nucleic Acids Res 1994, 22:4673-4680. 